\title{
Numerical test of the generalized Flory and generalized Flory dimer theories
}

\author{
G. C. A. M. Mooij and D. Frenkel \\ FOM Institute for Atomic and Molecular Physics, Kruislaan 407, 1098 SJ Amsterdam, The Netherlands
}

(Received 1 July 1993; accepted 22 December 1993)

\begin{abstract}
We report results of a numerical test of the generalized Flory and generalized Flory dimer theories. Using continuous space Rosenbluth sampling, we have tested the basic assumptions of these theories. We find that the discrepancy between the predictions of the generalized Flory theory and simulation data is caused by the use of the Carnahan-Starling equation of state to estimate the incremental chemical potential of a chain molecule. Although the assumptions made in deriving the generalized Flory dimer theory are similarly flawed, the predictions of the latter theory are in excellent agreement with simulation results. This is explained by the occurrence of a cancellation of errors. Therefore, it is not to be expected a priori that increasingly higher order extensions of these theories will be improvements. In particular, the disappointing performance of the recently developed generalized Flory trimer theory may be due to the fact that this theory lacks the cancellation of errors that is present in the generalized Flory dimer theory.
\end{abstract}

\section{INTRODUCTION}

Fifty years after its introduction Flory's theory ${ }^{1}$ is, no doubt, the most widely used theory to describe the statistical mechanics of polymeric systems. The theory is of mean-field character and was originally derived for lattice systems. It can account for many features of polymeric systems qualitatively, and in some cases even quantitatively: however, at least one empirical parameter (contained in the so-called $\chi$ parameter) has to be introduced to apply the theory to real continuous space systems and even to the lattice systems for which it was derived.

For lattice systems, the Flory theory models chains as a number of monomers occupying connected lattice sites. The same theory can also be derived for continuous space models. The predictions of the simple off-lattice version of Flory's theory are not in quantitative agreement with simulation results. ${ }^{2}$ Dickman and Hall ${ }^{2}$ showed that better results are obtained if the basic assumptions of Flory's theory for offlattice models are expressed in a more sophisticated manner. The approach of Dickman and Hall takes into account the essential differences between continuous space and lattice models and resulted in the so-called generalized Flory (GF) theory for chains of hard spheres. As shown in Ref. 2, the predictions of this theory and especially the extended version, the generalized Flory dimer theory ${ }^{3}$ (GFD), are in almost quantitative agreement with simulation results. As we will show below, this agreement is surprising in view of the rather severe approximations made in its derivation. The central quantity in the derivation of the GFD theory is the probability of successful insertion of a chain molecule in a fluid of such molecules. For hard-core systems this is equal to the probability that a molecule has no overlap with any other particle when inserted at random in the system. In order to test the basic assumptions of the GFD theory, one would like to calculate this insertion probability directly in a simulation. With conventional simulation techniques it is essentially impossible to insert chains longer than three to four monomers in a liquid in continuous space. Recently, however, we have developed a simulation technique, ${ }^{8}$ by means of which we can compute the insertion probability of longer chain molecules in a liquid. This makes it possible to test the basic assumptions of the GF and GFD theories directly. Here we present the results of these tests. First, we briefly describe the assumptions for the insertion probability in the GF and GFD theories. In Sec. III we explain the details of the model and present the simulation technique used. Finally we present the results of the simulations that we performed and compare with the assumptions of the GF and GFD theories.

\section{ASSUMPTIONS IN THE GENERALIZED FLORY (DIMER) THEORY}

Flory's theory ${ }^{1}$ for chain molecules was originally derived for a lattice model, in which the chains are modeled as a series of segments, occupying connected lattice sites. The derivation starts with an expression for the free energy of the system and from this expression all other thermodynamic functions are derived. An alternative route, presented by Dickman and $\mathrm{Hall}_{,}{ }^{2}$ starts from an expression for the insertion probability of a chain, and hence for the chemical potential. Thermodynamic consistency ensures that the thermodynamic functions derived from this expression are the same as the ones from Flory's original derivation. In order to obtain a tractable expression for $p(\eta, n)$, the insertion probability of a chain of $n$ monomers in a fluid of chains at a given volume fraction $\eta$, several assumptions are made. Flory's theory for lattice models can be obtained by ignoring all density correlations on the lattice and assuming

$$
p(\phi, n)=(1-\phi)^{n},
$$

where $\phi$ is the fraction of occupied sites on the lattice. A straightforward translation of this formula to a continuous space system with hard-core particles, is the replacement of $\phi$ by $\eta$, the volume fraction of the particles in the liquid. In that case, however, the accessible free space for monomers is overestimated. The idea in dexiving the generalized Flory theory is that the insertion probability for hard spheres in a 
hard-sphere fluid can be derived from the Carnahan-Starling equation. The first assumption then is that the insertion probability for a hard sphere in a hard-sphere fluid, $p_{1}(\eta, 1)$, is equal to that of a hard sphere in a fluid of hard-sphere chains of length $n$ at the same volume fraction $\eta, p_{n}(\eta, 1)$ :

$$
p_{n}(\eta, 1)=p_{1}(\eta, 1) \text {. }
$$

Another difference between lattice models and off-lattice models is that, in continuous space, the insertion probability for a hard-sphere chain of length $n$ will not be that for one hard sphere to the $n$th power. For a lattice model, this approximation [i.e., Eq. (1)] is correct if density correlations in the liquid are neglected. But for an off-lattice model, the equivalent of Eq. (1) will fail, even in the absence of density correlations. The reason is that for an $n$-mer in continuous space the excluded volume $v_{e}(n)$, i.e., the volume around the chain where no other hard sphere can be without having an overlap, is not $n$ times the excluded volume of a monomer $v_{e}(1)$, as it is on a lattice, but much less. From geometric arguments $v_{e}(2)$ can be calculated analytically and $v_{e}(3)$ can be calculated numerically. Extrapolation yields the following estimate for $v_{e}(n)$ for $n \geqslant 3$ :

$$
v_{e}(n)=v_{e}(3)+(n-3)\left[v_{e}(3)-v_{e}(2)\right] \text {. }
$$

With this assumption, we arrive at the GF prediction for $p_{n}(\eta, n)$ :

$$
p_{n}(\eta, n)=p_{n}(\eta, 1)^{v_{e}(n) / v_{e}(1)},
$$

where $p_{n}(\eta, 1)$ is estimated using Eq. (2).

It is possible to improve on the GF theory by exploiting the fact that, in addition to the Carnahan-Starling equation of state of hard spheres, we also have a good analytical approximation to the equation of state of hard dimers, the socalled Tildesley-Streett equation. ${ }^{5}$ As with the GF theory, we assume that the insertion probability for a hard dimer in a fluid of hard dimers, $p_{2}(\eta, 2)$, is equal to the insertion probability for a dimer in a fluid of $n$-mers, $p_{n}(\eta, 2)$ :

$$
p_{n}(\eta, 2)=p_{2}(\eta, 2) \text {. }
$$

From $p_{n}(\eta, 1)$ and $p_{n}(\eta, 2)$ an extrapolation to $p_{n}(\eta, n)$ can be made with the same excluded volume terms as before:

$$
p_{n}(\eta, n)=p_{n}(\eta, 1)\left[\frac{p_{n}(\eta, 2)}{p_{n}(\eta, 1)}\right]^{\left[v_{e}(n)-v_{e}(1)\right] /\left[v_{e}(2)-v_{e}(1)\right]} \text {. }
$$

This equation, together with Eqs. (2) and (5), form the GFD theory. The validity of the assumptions represented by Eqs. (2), (3), and (5), as well as the accuracy of the predictions found with Eqs. (4) and (6), can be tested by computer simulations. In Sec. III we describe the simulation technique which allows us to compute $p_{n}(\eta, n)$ at liquid densities.

\section{SIMULATION TECHNIQUE AND MODEL}

It is common in computer simulations to compute the insertion probability $p$ because it is directly related to the chemical potential $\beta \mu$ through Widom's expression: ${ }^{6}$

$$
\beta \mu^{\mathrm{ex}}=-\ln \langle p\rangle,
$$

where the brackets denote ensemble averaging. Using conventional sampling schemes, it is virtually impossible to compute the chemical potential for chains longer than three to four monomers in a liquid. The reason for this is that the insertion probability for these longer chains is so small, that it is impossible to successfully insert even a single chain during a simulation. In lattice systems, longer chains can be inserted by Rosenbluth sampling. ${ }^{7}$ Every new segment along a chain is not inserted at random on one of the sites adjacent to the position of the previous segment. Instead, it is determined by looking ahead, which of the sites are free and which are already occupied. If available, only free sites are sampled and thus occupied sites are avoided and holes in the system can be found. The bias introduced in this sampling procedure has to be corrccted for by assigning a Rosenbluth weight, $W$, to the configuration. In a lattice system with only excluded volume interactions this Rosenbluth weight is just the ratio of the number of available directions to the total number of trial directions. The simulation technique that we developed $^{8}$ extends this idea to continuous space. Because there is not a finite number of directions to choose from as on a lattice, we first choose a finite number of trial directions at random and then choose one of these according to the Rosenbluth procedure. In Ref. 8 it is shown that this is allowed for any number of trial directions. With this technique we can calculate the chemical potential by ${ }^{8}$

$$
\beta \mu^{\mathrm{ex}}=-\ln \langle W\rangle,
$$

where $\langle W\rangle$ is the ensemble average of the Rosenbluth weights, which is also equal to the average insertion probability $\langle p\rangle$. In this way, we can calculate insertion probabilities for longer chains to test the assumptions in the GF and GFD theories.

The model we use is the same as the one used in deriving the GF and GFD theories: "pearl necklace" 3 hard-sphere chains of length $n$, connected by freely rotating bonds which are of the same length as the diameter of the spheres $\sigma$. All simulations are done at a volume fraction $\eta=0.27$, typical for liquids. We calculated $p_{n}(\eta, n)$ for chains of lengths ranging from $n=1$ to $n=12$. The excluded volume of an $n$-mer, $v_{e}(n)$, for a hard sphere, is computed from the insertion probability $p$ for a hard sphere in a system with one isolated chain of $n$ monomers. This insertion probability is equal to the ratio of the free volume $v_{\text {free }}$ and the total volume $v_{\text {tot }}$. The excluded volume of the $n$-mer is given by

$$
v_{e}(n)=v_{\text {tot }}-v_{\text {free }}=(1-p) v_{\text {tot }} .
$$

\section{TEST RESULTS}

With the technique explained in Sec. III we have tested the assumptions that were described in Sec. II. We discuss the results in terms of the excess chemical potential, which is related to the insertion probability through Eq. (7) and which is calculated using formula (8). In Fig. 1 we have plotted the cxcess chcmical potential for a hard sphere in a fluid of chains of length $n, \beta \mu_{n}^{\mathrm{ex}}(\eta, 1)=-\ln p_{n}(\eta, 1)$, and in Fig. 1 the same for a hard dimer, $\beta \mu_{n}^{\text {ex }}(\eta, 2)=-\ln p_{n}(\eta, 2)$. The chemical potentials decrease appreciably with increasing $n$. This means that assumptions (2) and (5) are not valid, and 


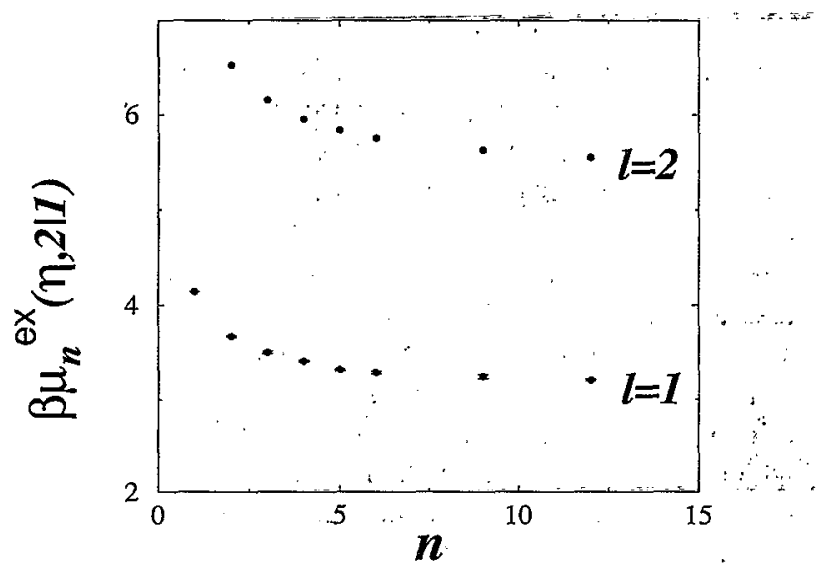

FIG. 1. The excess chemical potentials $\beta \mu_{n}^{\mathrm{ex}}(\eta, 1)$ and $\beta \mu_{n}^{\mathrm{ex}}(\eta, 2)$, respectively, for a hard sphere and a hard dimer in a fluid of chains of $n$ hard spheres at volume fraction $\eta=0.27$.

that the Carnahan-Starling and the Tildesley-Streett equations strongly overestimate the chemical potentials of a hard sphere and a hard dimer in a fluid of $n$-mers, respectively. In deriving the GFD theory, Honnell and $\mathrm{Hall}^{3}$ already pointed out that this could have been expected. With increasing $n$, more monomers cluster into $n$-mers, which causes an increase of the available space to insert a particle and as such a decrease of the excess chemical potential. We find that this is a significant effect. The second assumption is tested in Fig. 2, where the excluded volume for a hard-sphere chain of length $n, v_{e}(n)$, is shown. The fact that $v_{e}(n)$ depends linearly on $n$ shows that it can be estimated well by the extrapolation of the excluded volumes according to Eq. (3). This is in agreement with findings of Denlinger and Hall. ${ }^{9}$ They determined $v_{e}(n)$ by a numerical procedure similar to the one we use, and also drew the conclusion that Eq. (3) is a good estimate for the chain lengths considered in this paper. For longer chains they found a small deviation.

The final predictions of the GF and GFD theories are plotted in Fig. 3 and compared with our simulation results. In the same figure we show the prediction of the naive off-

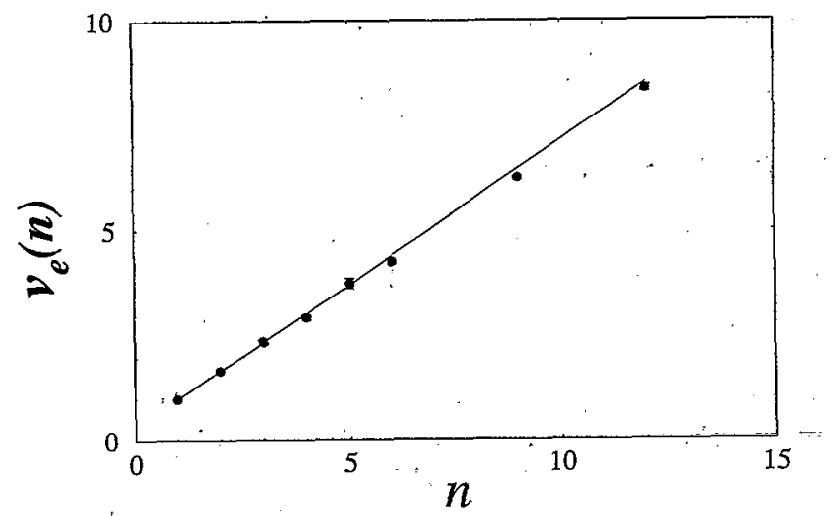

FIG. 2. The excluded volume of a chain of $n$ hard spheres, $v_{e}(n)$, for a hard sphere.

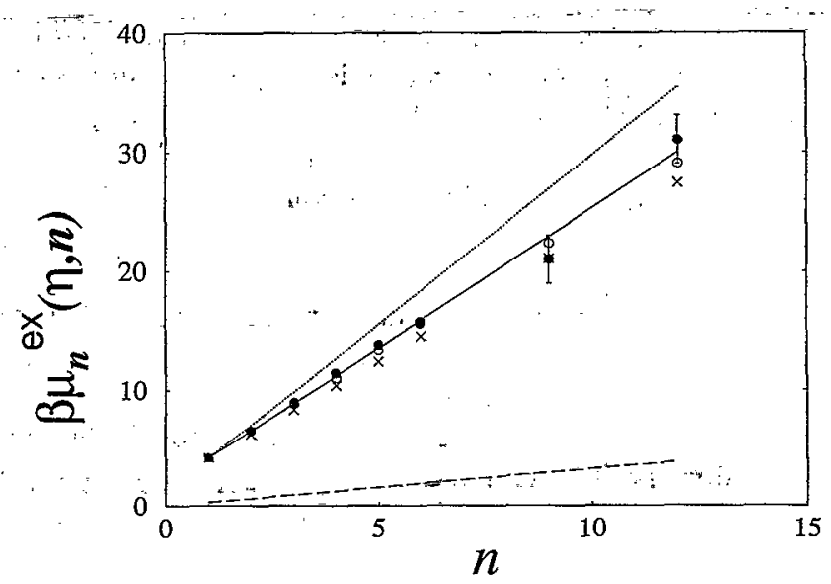

FIG. 3. The excess chemical potential $\beta \mu_{n}^{\mathrm{ex}}(\eta, n)$ for a chain of $n$ hard spheres in a fluid of $n$-mers at volume fraction $\eta=0.27$. : simulation results; --.: Flory's theory; $\cdots$ : GF theory; - - GFD theory; $X$ : GF theory with the true $\beta \mu_{n}^{\mathrm{ex}}(\eta, 1)$; and $\mathrm{O}$ : GFD theory with the true $\beta \mu_{n}^{\mathrm{ex}}(\eta, 1)$ and $\beta \mu_{n}^{\mathrm{ex}}(\eta, 2)$.

lattice version of Flory's theory, which underestimates the excess chemical potential strongly. This is explained by the fact that the straightforward translation of Flory's theory [Eq. (1)] to continuous space overestimates the available space to insert a particle. The GF theory does a much better job, but it results in an overestimate of the excess chemical potential. This becomes progressively worse with increasing chain length. It is a direct consequence of the fact that the theory does not account for the decrease of the chemical potential of a monomer in a fluid of $n$-mers (Fig. 1). Now one would expect that the GFD theory would not do any better, because an extra assumption [Eq. (2)] is made, which is no more valid than the one already made in the GF theory, as was shown in Fig. 1. We find, on the contrary, that the GFD theory agrees with our simulation data to within the statistical error of our calculations. That could happen only because of a cancellation of errors. The GFD theory contains the conditional probability $p_{n}(\eta, 2 \mid 1)=p_{n}(\eta, 2) / p_{n}(\eta, 1)$ [Eq. (6)], of inserting a second sphere after one sphere has been inserted without an overlap. For all $n$ larger than 2, both the numerator and the denominator in the conditional insertion probability for a second sphere are overestimated. Figure 4 shows that the net effect is a decrease of the residual excess chemical potential of the second segment, $\beta \mu_{n}^{\mathrm{ex}}(\eta, 2 \mid 1)$ $=-\ln p_{n}(\eta, 2 \mid 1)$, with increasing $n$, as can be expected again from the clustering of monomers into chains. But, by coincidence, the value for $\beta \mu^{\mathrm{ex}}(\eta, 2 \mid 1)$ that is used in the GFD theory is the same as the true one at $n=12$ (see Fig. 4). So, at the high $n$ in our range, the contributions of the residual excess chemical potentials of all segments except the first one, are estimated well. At $n=2$ the error in $\beta \mu_{n}^{\text {ex }}(\eta, 2 \mid 1)$ must be canceled exactly by the error in $\beta \mu_{n}^{\mathrm{ex}}(\eta, 1)$. At intermediate $n$ a combination of these effects occurs. Due to these effects the GFD theory predicts $\beta \mu_{n}(\eta, n)$ well over the range of values of $n$ that we have studied.

Until now, we have not considered the neglect of density 


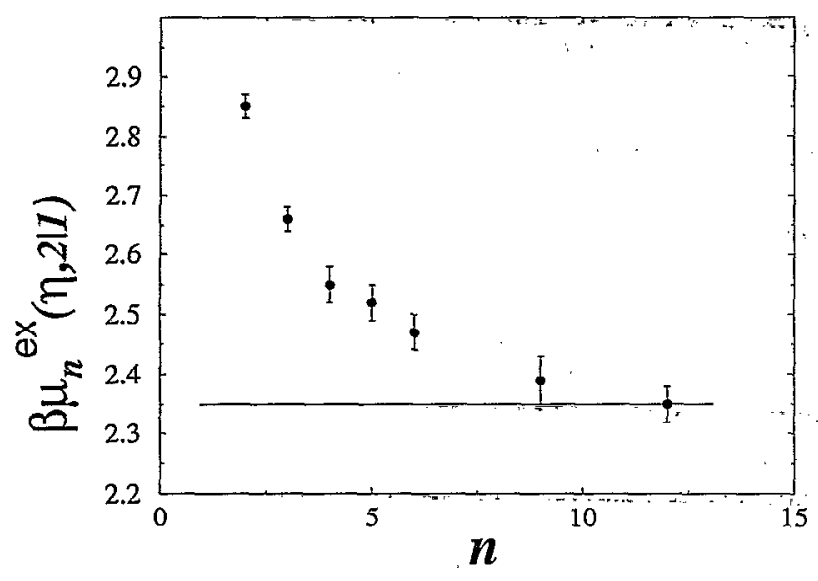

FIG. 4. The residual excess chemical potential $\beta \mu_{n}^{\mathrm{ex}}(\eta, 2 \mid 1)$ $=-\ln p_{n}(\eta, 2 \mid 1)$, where $p_{n}(\eta, 2 \mid 1)$ is the conditional probability for inserting a second hard sphere next to an already successfully inserted hard sphere. - : assumption for $\beta \mu_{n}^{\mathrm{cx}}(\eta, 2 \mid 1)$ in the GFD theory, using assumptions (2) and (5).

correlations in the fluid, which is intrinsic in Flory's derivation. If the actual value of $p_{n}(\eta, 1)$ would be known, the only approximation left in the GF theory is the neglect of all the density correlations. In Fig. 3 we show the values that we get for $\beta \mu_{n}(\eta, n)$ when we use the true $p_{n}(\eta, 1)$ from our simulation data (Fig. 1) in the GF cquation (4). Those valucs are close to, but a little below, the results of our simulations. This means that the remaining approximation, the neglect of density correlations, causes a small underestimate of $\beta \mu_{n}(\eta, n)$, which partly compensates for the overestimation, caused by assumption (2). If $p_{n}(\eta, 2)$ would be known too, the GFD theory would include density correlations on the dimer level, i.e., for the insertion probability of a monomer, $p_{n}(\eta, 1)$, and for the conditional probability of inserting a second sphere, $p_{n}(\eta, 2 \mid 1)$. Then the only approximation left is the assumption that the contribution of the correlations can be extrapolated to the remaining conditional probabilities $p_{n}(\eta, l \mid l-1)$ for $l>2$. In Fig. 3 we show the result of using the true $p_{n}(\eta, 1)$ and $p_{n}(\eta, 2)$ from our simulations in the GFD equation (6). It does not change the results, and the agreement with the simulation data remains excellent. Apparently, the contribution of density correlations to the conditional probability $p_{n}(\eta, 2 \mid 1)$ is the same as to the conditional probabilities $p_{n}(\eta, l \mid l-1)$ for $l>2$. It should be notcd that the simulations were performed at liquid densities and that for lower densities the situation might be different.

Recently, attempts have been made to improve the GFD theory. The GFD theory predicts the equation of state of $n$-mers by extrapolation from the monomer and dimer equations of state. Similarly, an extrapolation from the dimer and trimer equations of state can be used. This was done by $\because$ Yethiraj and Hall ${ }^{10}$ and the extended version is called the generalized Flory trimer theory (GFT). The predictions by the GFT theory are expected to be better, because GFT should incorporate the chain-like nature of the molecules more accurately. However, Yethiraj and Hall found that the performance of the GFD theory is superior to that of the GFT theory. Considering our test results for the GFD theory, this is not so surprising, because the cancellation of errors that occurs for the GFD theory need not be as complete in the GFT theory.

\section{CONCLUSIONS}

We have performed simulations to check the validity of assumptions made in deriving the generalized Flory and the generalized Flory dimer theory. We find that the CarnahanStarling equation does not yield an accurate estimate of the insertion probability of hard spheres in a fluid of chains of $n$ monomers, and leads to an overestimate of the chemical potential by the GF theory. The assumption in the GFD theory, that the Tildesley-Streett equation for hard dimers can be used for dimers in a fluid of chains of $n$ monomers, is not valid either, but the errors introduced by using both the Carnahan-Starling and Tildesley-Streett equations very nearly cancel.

The neglect of density correlations beyond the monomer level causes a small underestimate of the chemical potential by the GF theory, which partly compensates the overestimation caused by the use of the Carnahan-Starling equation. In the GFD theory density correlations are included up to the dimer level and further neglected, but this neglect has no effect on the results. In summary, the GF theory overestimates the chemical potential of an $n$-mer fluid and the GFD gives a good prediction of the chemical potential due to a cancellation of errors.

\section{ACKNOWLEDGMENTS}

The investigations reported in this paper were supported in part by "Scheikundig Onderzoek Nederland" (SON) with financial aid from "Nederlandse Organisatie voor Wetenschappelijk Onderzoek" (NWO). The work of the FOM Institute is part of the research program of FOM and is supported by NWO.

${ }^{1}$ P. J. Flory, I. Chem. Phys, 9, 660 (1941).

${ }^{2}$ R. Dickman and C. K. Hall, J. Chem. Phys. 85, 4108 (1986).

${ }^{3}$ K. G. Honnell and C. K. Hall, J. Chem. Phys. 90, 1841 (1989).

${ }^{4}$ N. F. Carnahan and K. E. Starling, J. Chem. Phys. 51, 635 (1969).

${ }^{5}$ D. J. Tildesley and W. B. Streett; Mol. Phys. 41, 85 (1980).

${ }^{6}$ B. Widom, J. Chem, Phys. 39, 2802 (1963).

${ }^{7}$ M. N. Rosenbluth and A. W. Rosenbluth, J. Chem. Phys. 23, 356 (1955);

J. Harris and S. A. Rice, ibid. 88, 1298 (1988); J. I. Siepmann and D. Frenkel, Mol. Phys, 75, 59 (1992).

${ }^{8}$ D. Frenkel, G. C. A. M. Mooij, and B. Smit, J. Phys. Condensed Matter 4, 3053 (1992).

${ }^{9} \mathrm{M}$. A. Denlinger and C. K. Hall, Mol. Phys. 71, 541 (1990).

${ }^{10}$ A. Yethiraj and C. K. Hall, Mol. Phys. 80, 469 (1993). 